\title{
A New Method for Vibration Suppression of Switched Reluctance Motor Using Liquid Cooling Medium
}

\author{
Luan $\mathrm{Ru}^{1, \text { * }}$, Bo Wei ${ }^{1,2}$ \\ ${ }^{1}$ College of Electrical and Information Engineering, Beijing University of Civil Engineering and Architecture, Beijing, China \\ ${ }^{2}$ Department of Industrial and Manufacturing Engineering, Florida State University, Tallahassee, United States
}

Email address:

luanru@bucea.edu.cn (Luan Ru), bw19k@my.fsu.edu (Bo Wei)

${ }^{*}$ Corresponding author

To cite this article:

Luan Ru, Bo Wei. A New Method for Vibration Suppression of Switched Reluctance Motor Using Liquid Cooling Medium. American Journal of Mechanical and Industrial Engineering. Vol. 6, No. 5, 2021, pp. 69-76. doi: 10.11648/j.ajmie.20210605.13

Received: September 7, 2021; Accepted: September 24, 2021; Published: September 29, 2021

\begin{abstract}
The vibration problem of a switched reluctance motor (SRM) comes from its structure and operation principle, and seriously affects its application. In fact, the vibration is caused by the radial electromagnetic force of SRM. The electromagnetic field numerical calculation software Magnet can simulate the direction of radial electromagnetic force. In addition, the Maxwell stress method was used to deduce the judgment rule of the direction of radial electromagnetic force in an experiment of measuring the flux distribution of SRM stator model. The finished test results confirmed the calculation results of Magnet software. On this basis, using the pressure of liquid cooling medium to reduce the radial electromagnetic force, a new type of switched reluctance motor stator structure is designed by immersing the whole stator with a moving fluid formed by the evaporative cooling liquid medium. It is proved by ANSYS and ANSYS Workbench software that under the effect of temperature difference, the evaporative cooling medium flows at a certain velocity field which strengthened the liquid pressure and the liquid pressure of the evaporative cooling medium counteract the radial electromagnetic force, and meanwhile weaken the vibration caused by it. As a conclusion, all the simulations show that the new type of SRM stator structure filled with evaporative cooling medium can reduce the SRM stator vibration speed by $40 \%$.
\end{abstract}

Keywords: Switched Reluctance Motor, Vibration, Radial Electromagnetic Force, Evaporative Cooling Medium, Stator, Simulation, LIQUID Pressure

\section{Introduction}

A switched reluctance motor (SRM) depends on the change of the reluctance, that is, the SRM with a strong nonlinear switching power supply and a doubly salient structure which leads to nonlinear current, can change the reluctance of the motor, and then produce the driving torque. Moreover, the change of reluctance not only produces the tangential electromagnetic pull, but also the radial pulsating electromagnetic suction, which tries to attract the stator and rotor to each other and compress the air gap between them. The theoretical study shows that the radial electromagnetic force of SRM is far greater than its driving force. Under the action of such force field, the rotor is a solid cylinder with good rigidity, which is basically not affected by the radial electromagnetic force; while the stator is a shell structure, which inevitably produces regular compression and expansion vibration due to the radial electromagnetic force, and the vibration emits noise through the shell [1-3]. The experimental results show that the SRM has the most serious vibration and noise among all driving motors, which seriously limits its application $[4,5]$.

Since the vibration and noise are the structural electromagnetic vibration and noise of SRM, many researchers have thought to solve the vibration problem from its structure. For example, around the stator structure, some scholars have proposed the stator structure which is the result of optimization with the objective of reducing vibration displacement and noise, however it is only effective in a certain medium and high frequency band [6]. The structure of rotor teeth and air gap have the same influence on SRM vibration. For example, some scholars propose to cut grooves on the rotor teeth to improve the inductance variation curve [7], or change the shape of the inner pole of the rotor to form 
an asymmetric air gap distribution [8], which can reduce the vibration, but these changes on the rotor teeth increase the magnetic flux leakage and reduce the output of the motor.

Researchers also continue to design new structures of SRM [9]. For example, a multi pole multi segment rotor and axial flux structure [10]. The research shows that the axial flux component of this structure accounts for more than $70 \%$ of the total flux, which greatly improves the power density, and the torque increases by $40 \%$ compared with the conventional structure. At the same time, the radial electromagnetic force which causes vibration and noise are reduced due to the reduction of radial flux, but its fatal weakness is that the stator winding loss increases significantly, the leakage flux through the casing also increases significantly, and then the efficiency decreases. Similarly, Matsui, et al. [11] proposes a structure that the phases of SRM are arranged separately along the axis. With the increase of the number of poles in each phase, the torque increases and the radial electromagnetic force decreases slightly, but the power density does not increase and the stator winding loss increases significantly.

It is assumed that the switched reluctance motor operates in the single-phase conduction state, and the radial electromagnetic force is larger when the conduction is disconnected. The air damping is very small. Obviously, if the damping factor is not taken into account, the vibration amplitude is proportional to the radial electromagnetic force according to the classical Newton's law of mechanics. If a new stator structure to counteract the effect of radial electromagnetic force on the stator pole is tried, the amplitude and vibration acceleration will be significantly reduced accordingly [12].

The problem is how to counteract the effect of the radial electromagnetic force on the stator pole without affecting the performance, volume, efficiency and other important indexes of the motor? In this paper, a perfusion structure, can counteract the effect of the radial electromagnetic force on the stator pole. The so-called perfusion structure refers to the immersed evaporative cooling stator structure [13]. If the stator sealing cavity is filled with liquid evaporative cooling medium with high specific gravity and viscosity, the liquid is not only a damping medium (compared with air), but also the insulation medium or cooling medium. Because the heating body of SRM is mainly stator, evaporative cooling with the best cooling effect can completely replace fan cooling, so as to remove the fan and eliminate the aerodynamic noise caused by the fan. Using the immersed evaporative cooling stator structure, the current density of stator winding and the magnetic density in the core can be slightly higher as the water cooling method, so as to reduce the volume of switched reluctance motor and significantly improve the power density. It can be seen that the cooling, insulation and vibration suppression can all be solved by the structure, and the performance index of SRM can be improved.

\section{Action Direction of Radial Electromagnetic Force in SRM}

Because of the switching power supply with strong nonlinearity and the doubly salient structure which leads to nonlinear current, the calculation of the radial electromagnetic force of SRM can only be realized by the magnetic field numerical analysis method [14], and then the experimental model is used to test. In order to explain the research process, this paper is based on a $5 \mathrm{~kW}, 12$ / 8 pole switched reluctance motor.

\subsection{Numerical Analysis of Radial Electromagnetic Force}

In this paper, the Magnet software in the professional software package Infolytica 7.0 is used to model the two-dimensional field of the $5 \mathrm{~kW}, 12$ / 8-pole SRM, that is, the cross section perpendicular to the axial direction of the motor is taken, including the stator core and its windings, air gap and rotor core. Under the excitation of single-phase conduction, the transient magnetic field of the two-dimensional model as shown in Figure 1 is calculated, and the distribution of the radial electromagnetic force at a certain moment is obtained. In order to see the direction of the radial electromagnetic force on the stator side clearly, the local stator area is enlarged in Figure 2. It can be seen from the figure that the direction of radial electromagnetic force acting on the stator is from the solid surface of the stator to the outside, and the direction of action will not change with the speed, but the radial electromagnetic force will fluctuate with the change of rotor position, which is consistent with the principle of radial electromagnetic suction.

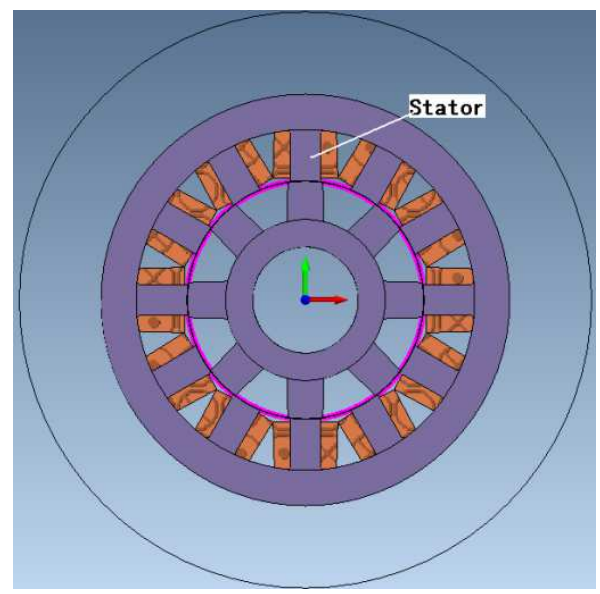

Figure 1. Calculation results of radial electromagnetic force in two-dimension field.

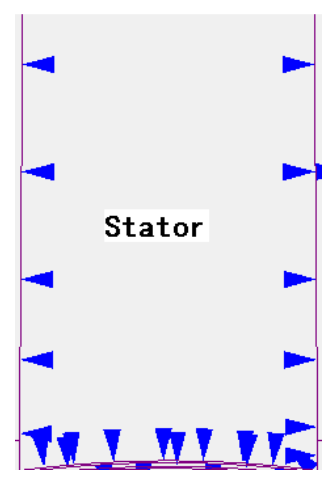

Figure 2. Amplified radial electromagnetic force on stator. 


\subsection{Experimental Measurement of Radial Electromagnetic Force Direction}

The direction of the radial electromagnetic force can be obtained not only by the above numerical calculation method, but also by the appropriate experimental measurement method, which further confirms the direction of the radial electromagnetic force. Direct measurement is impossible, but the formula of radial electromagnetic force can be deduced by using Maxwell stress method. For the calculation model of SRM shown in Figure 1, the following reasonable assumptions are made: (1) the magnetic vector potential $\vec{A}$ and stator current only have $z$ axial component; (2) the physical quantities related to the magnetic field distribution do not change along the axial direction. According to the physical meaning of magnetic vector potential $\vec{A}$ and Maxwell's equations, there is a conclusion below:

$$
\begin{gathered}
\vec{B}=\nabla \times \vec{A} \\
\nabla \times\left[\frac{1}{\mu_{0}}(\nabla \times \vec{A})\right]=\vec{J}_{0}
\end{gathered}
$$

Where $\vec{B}, \vec{J}_{0}$ and $\mu_{0}$ are the field flux density, the stator current density and air permeability respectively. According to the theory of electromagnetic field, the formula of the electromagnetic force density acting on the object in the magnetic field is:

$$
\vec{f}=\vec{J}_{0} \times \vec{B}
$$

Another algorithm of electromagnetic force density can be obtained by taking equations (1) and (2) into equation (3):

$$
\vec{f}=\frac{1}{\mu_{0}}(\nabla \times \vec{B}) \times \vec{B}
$$

Then the electromagnetic force acting on the object of volume $V$ is:

$$
F=\int_{V} f d V=\int_{V} \frac{1}{\mu_{0}}(\nabla \times B) \times B d V
$$

According to the vector operation, there is the following formula:

$$
(\nabla \times B) \times B=(\nabla \bullet B) B-\frac{1}{2} \nabla B^{2}
$$

Take equation (6) into equation (5) to get equation (7).

$$
F=\int_{S} \frac{1}{\mu_{0}}\left[(B \bullet \hat{n}) B-\frac{1}{2} \nabla B^{2} \hat{n}\right] d S
$$

When using the finite element method to calculate equation (7), let the solution domain be discretized into $m$ elements, then the electromagnetic force $\boldsymbol{F}$ of equation (7) is
$F=\sum_{e=1}^{m} F^{e}, F^{e}$ is the force per element. Assuming the area of each element is $\Delta S$, the tangential component $F_{x}^{e}$ and the normal component $F_{y}^{e}$ of the electromagnetic force in each element are derived according to the discrete algorithm of the finite element method. The tangential component is along the tangential direction of the action surface, corresponding to the torque direction of the SRM. The normal component is along the normal direction of the action surface, corresponding to the radial electromagnetic force of the SRM. They are equation (8) and (9) respectively.

$$
\begin{aligned}
& F_{x}^{e}=\frac{\Delta S}{\mu_{0}} B_{x} B_{y} \\
& F_{y}^{e}=\frac{\Delta S}{\mu_{0}}\left(B_{y}^{2}-B_{x}^{2}\right)
\end{aligned}
$$

If the area of each element is small enough, $F_{y}^{e}$ becomes the radial electromagnetic force at a point. If $B_{y}^{2}$ is greater than $B_{x}^{2}$, the radial electromagnetic force at that point is the same as the normal direction; if $B_{y}^{2}$ is less than $B_{x}^{2}$, the radial electromagnetic force at that point is opposite to the normal direction. Therefore, by measuring $\vec{B}$ at each sampling point in the study area, according to the size of $B_{y}$ and $B_{x}$ at each sampling point, the fact whether the direction of radial electromagnetic force at each point is outward or inward along the normal direction of the stator surface can be judged. According to this conclusion, the local model of the $5 \mathrm{~kW}, 12$ / 8 pole SRM has been designed and made with the ratio of $1: 1$, as shown in Figure 3.

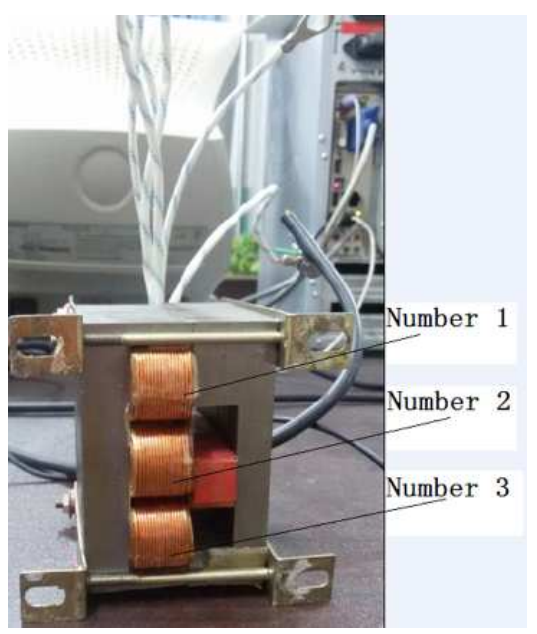

Figure 3. Model of partial section of $5 K W, 12 / 8$ poles SRM.

This experimental process, analysis and conclusion have been described in the paper (reference 15) published by the author of this paper, and so will not be repeated here. The conclusion of this experiment is that the radial 
electromagnetic force on the stator pole points outward from the surface of the stator pole, which is consistent with the calculation result of Magnet software described in Section 2.1.

\section{A New Method of Suppressing Vibration Caused by Radial Electromagnetic Force}

The direction of the radial electromagnetic force of SRM stator is obtained, which provides a guarantee for applying a force opposite to the direction, so as to counteract the radial electromagnetic force and reduce the vibration caused by it.

\subsection{Force Analysis of Stator Surface Immersed in Liquid}

First of all, according to the basic knowledge of hydrostatics, the hydrostatic pressure on any space wall is equal to the product of its submerged depth, liquid density and gravitational acceleration. Its acting direction is along the normal direction of the acting surface and points inward, which is opposite to the radial electromagnetic force obtained in the previous study. Therefore, the liquid pressure can be used as the reverse force of the radial electromagnetic force to eliminate its effect. Moreover, when the liquid moves, according to the knowledge of fluid dynamics, there is the following formula:

$$
\frac{\partial p}{\partial t}=v_{x} \frac{\partial p}{\partial x}+v_{y} \frac{\partial p}{\partial y}+v_{z} \frac{\partial p}{\partial z}+\frac{\partial p}{\partial t}
$$

$v_{y}$ Where $p$ is the pressure of the fluid, $v_{x}, v_{\mathrm{y}}, v_{z}$ are the velocities of the fluid in the three-dimensional direction of space. It can be seen that in the moving liquid, the liquid pressure on the internal solid surface will increase compared with the liquid in the static state, and the increase amplitude is proportional to the liquid velocity and its change rate with the pressure. If the stator is immersed in the moving insulating liquid, the counteracting effect of the liquid pressure on the radial electromagnetic force will be further strengthened. These physical laws have laid a foundation for the rationality and feasibility of the new method proposed in this paper.

\subsection{Evaporative Cooling Sealing Structure of SRM Stator}

In order to immerse the stator of SRM in insulating cooling liquid, in this paper, a new type of stator structure is designed, that is, the stator is completely sealed. However, this sealing structure is difficult to achieve. According to the previous stator sealing structure, it needs to place a sealing cylinder along the inner surface of the stator in the air gap between the stator and rotor to wrap the stator, but the sealing cylinder is not suitable for SRM. Because the SRM uses the change rate of the main flux (also known as the reluctance) between the stator and rotor to generate the electromagnetic torque to drive the motor rotation, its air gap is very small, and nothing can be placed. If the air gap is increased in order to place the sealing cylinder, the change rate of the main flux and the main flux will be reduced, but the leakage flux will be increased, which will weaken the driving torque and increase the noise. It is necessary to redesign the method of sealing SRM stator structure. Let's take a look at the stator of SRM, as shown in Figure 4. The stator winding is tightly wound on the magnetic pole of the stator core, and the magnetic pole is completely wrapped, only the inner surface of the magnetic pole of the stator core is exposed. In the stator slot between the magnetic poles, the stator winding wound on the magnetic pole is tightly pressed with slot wedge, so that the stator winding can be reliably fixed in the stator slot. After installation, the inner surface of the whole stator is composed of the inner surface of the magnetic pole of the stator core and the stator slot wedge, which is a closed polyhedron shape.

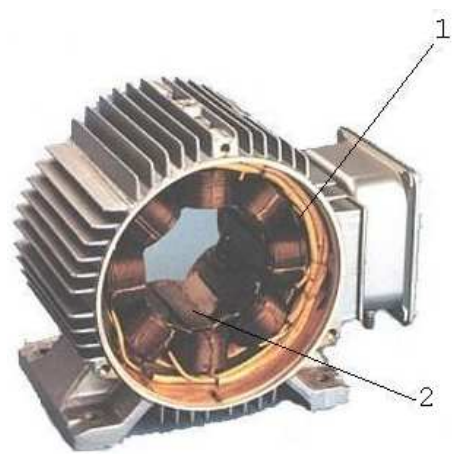

Figure 4. Stator in SRM: 1. Stator winding wound on the magnetic pole of stator core; 2. Slot wedge for fixing stator winding.

The new sealing structure in this paper makes full use of the original structure of SRM without changing its air gap. The design method of the new structure is as follows. Instead of slot wedges, the insulating board shown in Figure 5 is pressed onto the stator winding. The insulating board is composed of two parts. The part used for pressing stator winding in slot has the same function as the original slot wedge. Its length $\mathrm{L}$ is half of the length of stator core, and its width $d$ is the width of original slot wedge. The other part is used for sealing the end of stator core and winding, and its length L1 is based on the length of SRM end (specify the distance between the end of stator core and the end cover of motor casing). While the width $\mathrm{d} 1$ is closely related to the distance between the two adjacent stator core poles. Press the two insulating boards on the stator winding in the slot with tools from both sides of the stator core slot to ensure that the insulating board is in close contact with the stator core pole without leaving any gap. After the completion, there are two insulating boards for sealing in each slot, which occupy half of the space respectively. The inner surface of the stator is composed of the inner surface of the stator core pole and the surface of the insulating board for pressing the stator winding, which is a closed polyhedron shape. The two ends of the core are L1 parts of the insulation board with more axial length than the core, and there is a large overlap between these insulation boards. Bend, cut and shape these L1 parts of the insulation board at both ends along the circumferential radian of their respective positions to form a cylindrical shape, and then they 
are drilled together, as shown in Figure 6. The transverse section of the whole sealing structure effect is shown in Figure 7, which separates the inside and outside of the stator into two spaces.

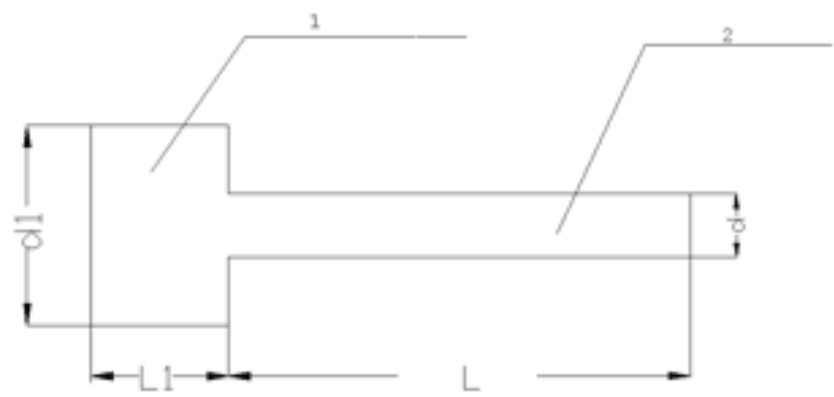

Figure 5. Insulating board for sealing: 1. The part used for sealing the end of stator core and winding; 2. The part Used for pressing stator winding in slot.

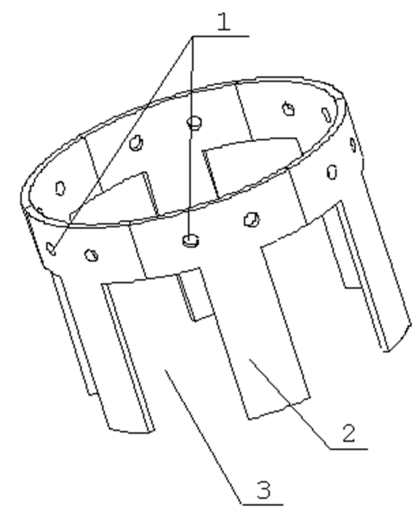

Figure 6. The sealing insulating board in the shape of a cylinder: 1. Drilling with each other; 2. The part Used for pressing stator winding in slot; 3. Location of inner surface of the magnetic pole of stator core.

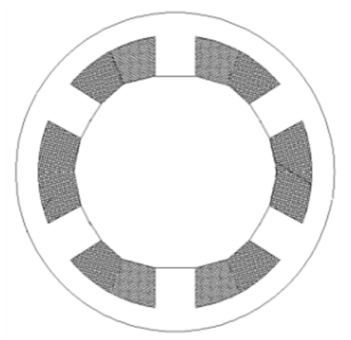

Figure 7. Cross section of the new sealing structure used in the SRM stator.

Next, the end connection structure shown in Figure 8 is used to connect the insulating board shown in Figure 6 with the housing of SRM. Then heat and dip paint the whole SRM body (no rotating part) in high vacuum state repeatedly until the inner surface of the stator cavity formed by the inner surface of the stator core pole and the insulating board is a complete, solid and highly sealed polyhedron. Then, a condenser is installed on the upper part of the stator sealing cavity, and the evaporative cooling medium is poured into the stator sealing cavity, as shown in Figure 9. When the motor is running, the heat from the stator core and winding is transferred to the evaporative cooling medium. After the medium boils to a gaseous state, it rises due to the decrease of density and contacts with the condensing tube. After the heat is transferred to the condensing tube, it is condensed back to the liquid state. In this way, the heat from the stator can be continuously transferred to the condenser, and then all the heat is dissipated through the condenser. In this way, the SRM stator is placed in the liquid to bear the liquid pressure, and the liquid can quickly form a certain speed of fluid after being heated. Therefore, using this method of evaporative cooling the stator, liquid pressure can be generated to reduce the radial electromagnetic force that causes SRM vibration.

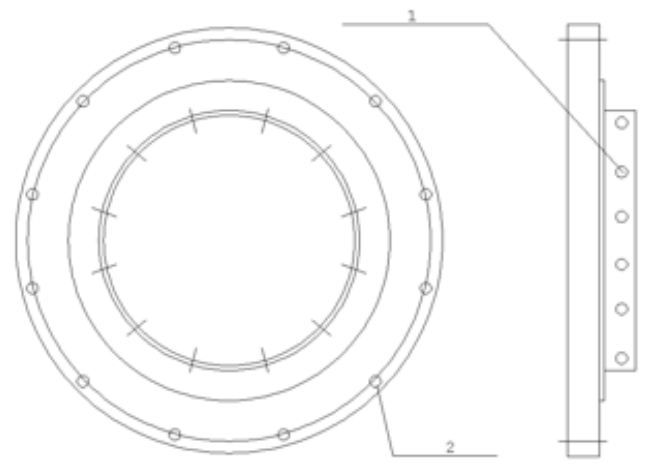

Figure 8. End connection structure: 1. Holes for connection with the insulating board; 2. Holes for connection with the housing.

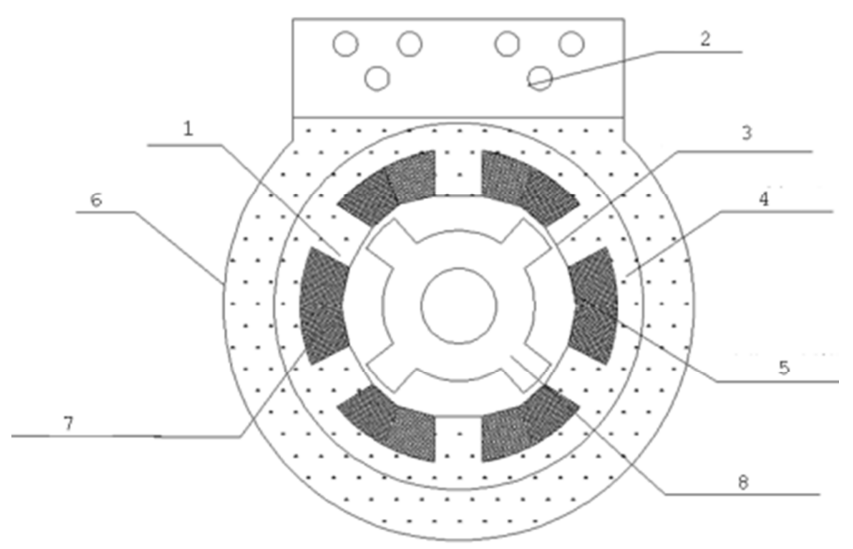

Figure 9. Stator immersed in evaporative cooling medium and its sealing structure: 1. Magnetic pole of stator core; 2. Condenser; 3. The inner surface of the magnetic pole of the stator core; 4. Evaporative cooling medium; 5. Insulation board for sealing; 6. Motor housing; 7. Stator slot and stator winding; 8. The magnetic pole of the rotor core (there is no winding on the rotor).

\section{Simulation Results of the New Method Suppressing Vibration and Discussion}

In order to understand and verify that the structure of evaporative cooling stator shown in Figure 9 can reduce the vibration of SRM, two mathematical models are established for the structure and the fluid field and transient structure field are calculated respectively.

\subsection{Fluid Field Simulation Results of Evaporative Cooling Stator Structure and Discussion}

The preliminary numerical simulation of fluid field is carried 
out by using ANSYS 8.0 software, as shown in Figure 10. The calculation is still based on the $5 \mathrm{~kW}, 12$ / 8 pole SRM. The inner circle in the figure represents the inner surface of the stator sealing body, and the average operating temperature is set at $50^{\circ} \mathrm{C}$ (The boiling point of the evaporative cooling medium is $47^{\circ} \mathrm{C}$, and the temperature of the evaporative cooling medium in the SRM stator sealing cavity can be controlled within $50^{\circ} \mathrm{C}$ ). The outer circle is the casing, and the evaporative cooling medium is filled between the inner and outer circles, indicating that the whole stator is immersed in the evaporative cooling medium. If the initial temperature of the cooling medium is set at $30^{\circ} \mathrm{C}$, the simulation results show that the evaporative cooling medium can quickly establish a certain scale velocity field driven by the temperature difference between the stator heat generator and the medium. If the liquid flow intensified by bubbles in the process of medium boiling and vaporization is considered, the velocity field is strengthened with the continuous operation of the SRM.

It can be seen that the SRM stator immersed in evaporative cooling liquid bears static pressure caused by liquid gravity on the one hand, and dynamic pressure caused by moving fluid velocity on the other hand. According to the reasoning analysis in Section 2 above, the liquid pressure and the radial electromagnetic force acting on the stator are in the opposite direction, counteracting each other, thus weakening the force of SRM vibration.

\subsection{Simulation for Transient Structural Field of Evaporative Cooling Seal Body and Discussion}

As everyone knows, there must be a reference to compare the strength of a vibration phenomenon. Therefore, in this paper the conventional stator structure is chosen as the reference to compare with the evaporative cooling stator structure. For SRM, the source of its strong vibration is the radial electromagnetic force, which needs to use the finite element method of transient structural field to solve its vibration velocity. In this paper, ANSYS Workbench 14.5 software is used to calculate the transient structure field in the evaporative cooling stator seal body and the transient structure field of conventional stator under the same excitation source and boundary conditions. The vibration velocities of these two structures are obtained, which are shown in Figure 11 for comparison.

Due to the shell structure of the stator, the stator with conventional structure will vibrate strongly as long as it is subjected to electromagnetic force, and the maximum vibration speed reached $82.7 \mathrm{~mm} / \mathrm{s}$. Because the rotor is fixed on the shaft, it is a solid cylinder, and the vibration speed is very low, which can be ignored compared with the stator. This result is basically consistent with the vibration phenomenon in the actual SRM. When the stator is immersed in the flowing evaporative cooling medium, the vibration speed of the stator decreases by nearly half, and the maximum vibration speed is $49.9 \mathrm{~mm} / \mathrm{s}$, which indicates that the introduction of the medium liquid into the stator vibration system indeed weakens the vibration intensity, which confirmed the research results in Section 2 above. As the liquid pressure increases with the increase of liquid height and liquid flow velocity, the liquid height at the bottom of the stator seal body is the highest. As can be seen from Figure 11, the liquid flow velocity here is also the highest. As a result, the pressure at the bottom of the stator is significantly higher than that at the top, and the liquid pressure at the bottom counteracts the radial electromagnetic force to a greater extent, causing the vibration at the top of the SRM to be significantly stronger than that at the bottom, which further proves that the liquid pressure can counteract the radial electromagnetic force.

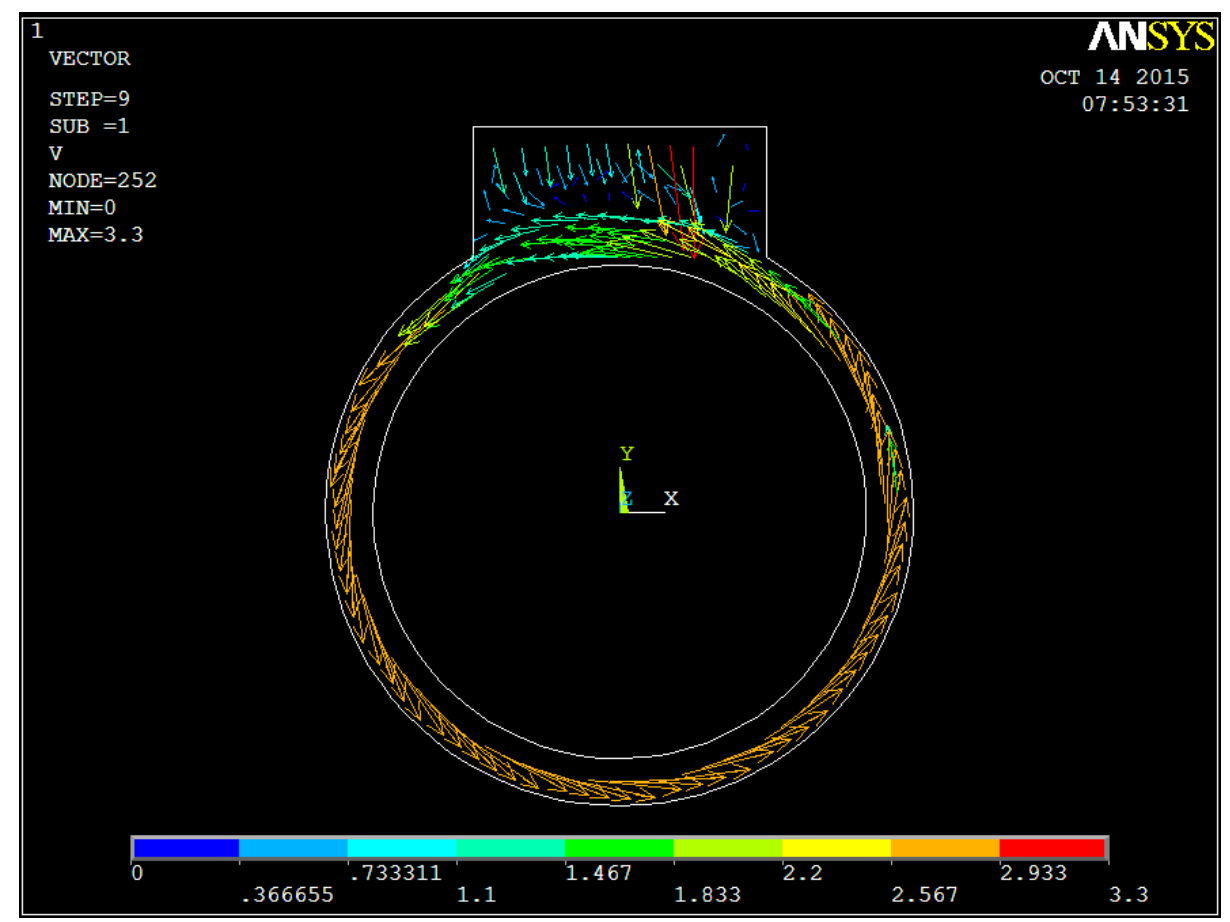

Figure 10. Model of partial section of $5 K W, 12 / 8$ poles SRM. 


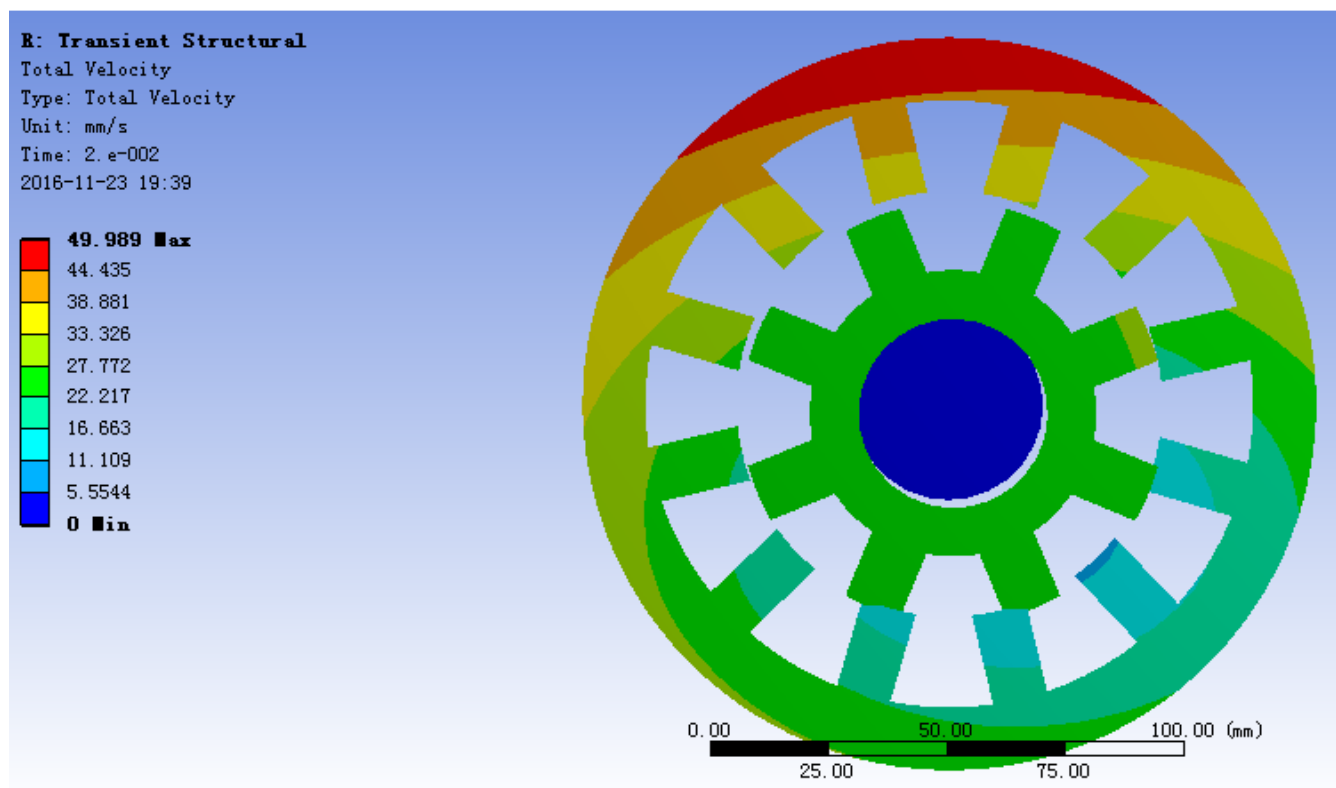

(a)

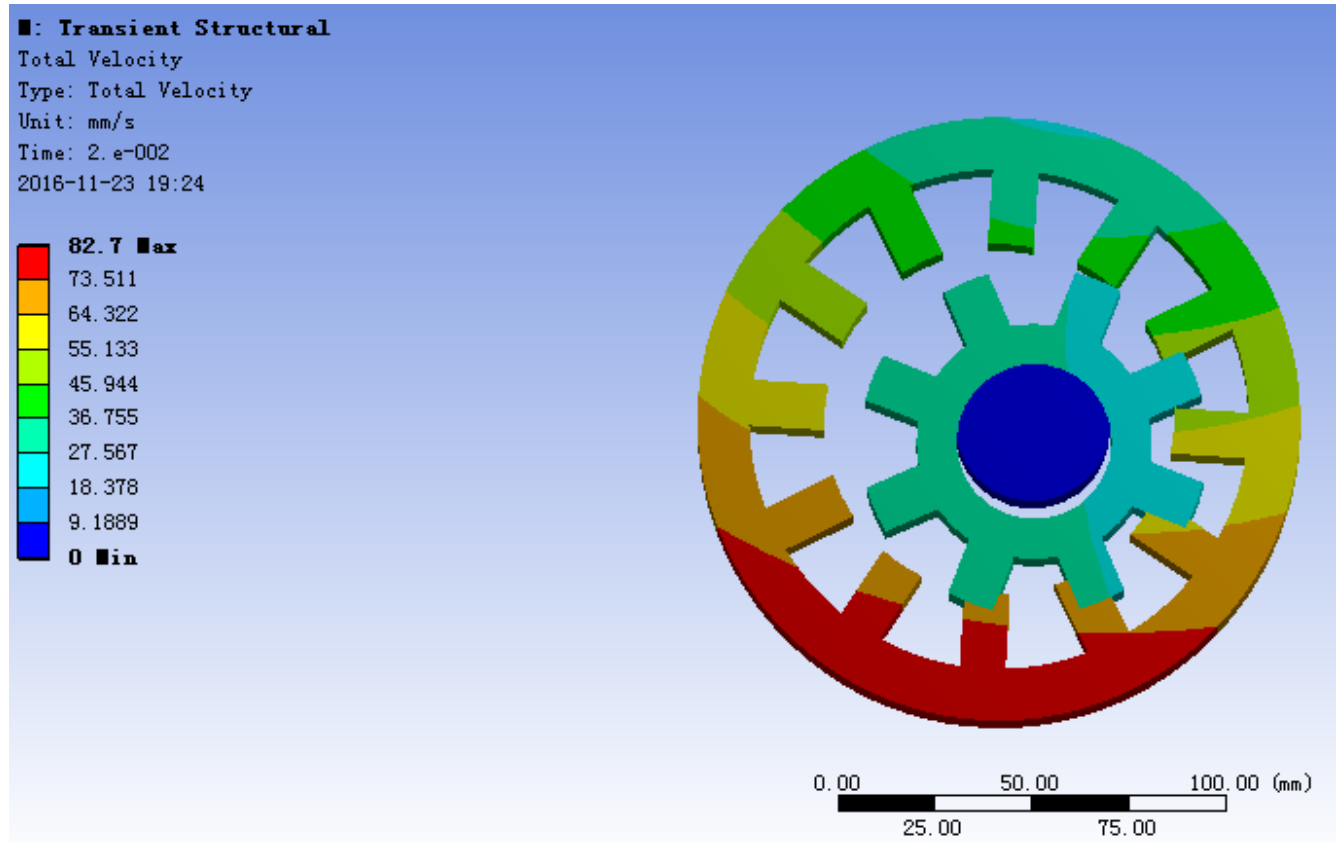

(b)

Figure 11. Comparison of vibration velocity of two transient structural fields at the same time, they are: (a) The conventional stator of SRM; (b) The evaporative cooling stator of SRM.

\section{Conclusions}

According to the results of numerical simulation and measurement, the radial electromagnetic force distribution of the stator is opposite to the direction of the liquid pressure. It is concluded that these two forces can counteract each other, and then the method of using evaporative cooling liquid to mitigate the stator vibration of SRM is proposed. Specifically, the whole SRM stator is completely sealed, the evaporative cooling medium is poured into the stator sealing cavity, and a condenser is installed on the top of the SRM stator, so that the liquid with density greater than water is filled between the stator and the casing. The heat generated during SRM operation heats the evaporative cooling medium, makes it flow continuously, and further increases the pressure of the medium liquid. The liquid pressure of evaporative cooling medium weakens the source of SRM vibration, which has been realized by simulation with ANSYS Workbench 14.5 software. The results show that the sealing structure filled with evaporative cooling medium can reduce the SRM stator vibration speed by $40 \%$. And because the evaporative cooling 
medium has no effect on the magnetic field distribution, the output performance of the SRM will not be affected.

Therefore, the evaporative cooling stator structure proposed in this paper will provide a feasible and reasonable technical solution for SRM vibration problem.

\section{Acknowledgements}

This research was funded by the Fundamental Research Funds for Beijing Universities, grant number FZ04.

\section{References}

[1] D. E. Cameron, J. H. Lang and S. D. Umans, (1992). The origin and reduction of acoustic noise in doubly salient variable reluctance motor, IEEE Transactions on Industry Applications, 28 (6): 1250-1255.

[2] K. Kiyota, T. Kakishima, H. Sugimoto, A. Chiba, (2013). Comparison of the Test Result and 3D-FEM Analysis at the Knee Point of a $60 \mathrm{~kW}$ SRM for a HEV, IEEE Transactions on Magnetics, 49 (5): 2291- 2294.

[3] Wu Jianhu, (2004). Study on the stator mode shape and natural frequencies of switched reluctance motor based on real structural mode. Proceedings of the CSEE, 24 (8): 109-114. (in Chinese).

[4] C. Pollock and W. Chiyao, (1997). Acoustic noise cancellation techniques for switched reluctance drives, IEEE Transactions on Industry Applications, 33 (2): 477-484.

[5] WANG Hong-hua, WANG Zhi-ping, JIANG Quan, (2005). Analytical Calculating of Natural Frequencies of Stator of Switched Reluctance Motor Based on Electromechanical Analogy Method. Proceedings of the CSEE, 25 (12): 133-137.

[6] Kamran Masoudi, Mohammad Reza Feyzi, and Amir Masoudi. (2013). Reduction of Vibration and Acoustic Noise in the Switched Reluctance Motor by Using New Improved Stator yoke shape, 978-1-4673-5634-3/13/\$31.00 (2013 IEEE, 1-4.
[7] Zhang Xin, Wang Xiuhe, Yang Yubo (2015). The Computation of Vibration Reduction Capacity for Switched Reluctance Motor Based on Improved Magnetic Field Partition Method, Transactions of China Electrotechnical Society, 30 (22): 9-18.

[8] Y. K. Choi, H. S. Yoon, et C. S. Koh, (2007). Pole-shape optimization of a switched-reluctance motor for torque ripple reduction, Magn. IEEE Trans. On, 43 (4): 1797-1800.

[9] Chun Gan, Jianhua Wu, Mengjie Shen, and etc. (2015). Investigation of Skewing Effects on the Vibration Reduction of Three-Phase Switched Reluctance Motors, IEEE Transactions on Magnetics, 51 (9): 1-10.

[10] Vandana, R.; Fernandes, B. G. (2015). Design Methodology for High-Performance Segmented Rotor Switched Reluctance Motors [J], Energy Conversion, IEEE Transactions on, 30 (1): 11-21.

[11] Matsui, Noriya Nakao, Kan Akatsu. (2014). Torque/Current Ratio Improvement and Vibration Reduction of Switched Reluctance Motors Using Multi-stage Structure [J], IEEE The 2014 International Power Electronics Conference, 1128-1134.

[12] Majid Asgar and Ebrahim Afjei, (2016). Radial Force Reduction in a New Flat-Type Double-Stator Switched Reluctance Motor [J], IEEE Transactions on Energy Conversion, 31 (1): 141-149.

[13] Luan $\mathrm{Ru}$, (2009). Insulation and Heat Transfer of a Horizontal Evaporative Cooling motor stator, Beijing: Science Press.

[14] Xiaobin Liang, Guangjin Li, Javier Ojeda, Mohamed Gabsi, and Zhuoxiang Ren, (2014). Comparative Study of Classical and Mutually Coupled Switched Reluctance Motors Using Multiphysics Finite-Element Modeling [J], IEEE Transactions on Industrial Electronics, 61 (9): 5600-5074.

[15] $\mathrm{Ru}$ Luan, (2017). Comparison of vibration between an evaporative cooling switched reluctance motor and a conventional switched reluctance motor" [J], IEEE 58th International Scientific Conference on Power and Electrical Engineering of Riga Technical University (RTUCON), 2017, October, 1-4. 